Center

for

Economic Research

No. $2000-51$

\title{
OPTIMAL TAX DEPRECIATION UNDER A
}

PROGRESSIVE TAX SYSTEM

By Jacco L. Wielhouwer, Anja De Waegenaere and Peter M. Kort

May 2000

ISSN 0924-7815 


\title{
Optimal tax depreciation under a progressive tax system
}

\author{
JACCO L. WielhouWer* ${ }^{*}$ Anja De WAEgenaere ${ }^{\dagger}$ \\ PETER M. KORT ${ }^{\ddagger}$
}

May 9, 2000

\begin{abstract}
The focus of this paper is on the effect of a progressive tax system on optimal tax depreciation. By using dynamic optimization we show that an optimal strategy exists, and we provide an analytical expression for the optimal depreciation charges. Depreciation charges initially decrease over time, and after a number of periods the firm enters a steady state where depreciation is constant and equal to replacement investments. This way, the optimal solution trades off the benefits of accelerated depreciation (because of discounting) and of constant depreciation (because of the progressive tax system). We show that the steady state will be reached sooner when the initial tax base is lower or when the discounting effect is stronger.
\end{abstract}

Keywords: tax depreciation, progressive tax system, discounting, dynamic optimization, path coupling.

\footnotetext{
${ }^{*}$ CentER, Tilburg University, P.O. Box 90153, 5000 LE Tilburg, The Netherlands.

${ }^{\dagger}$ CentER, Tilburg University, P.O. Box 90153, 5000 LE Tilburg, The Netherlands.

${ }^{\ddagger}$ Corresponding author: CentER and Department of Econometrics, Tilburg University, P.O. Box 90153, 5000 LE Tilburg, The Netherlands.
} 


\section{Introduction}

Since taxable income consists of gross revenue reduced with depreciation charges, the depreciation method that is chosen for tax purposes can be used as a strategic tool to allocate taxable income over future periods (see e.g. Scholes and Wolfson, 1992). Firms often have the option to choose between one or more accelerated methods, which have decreasing depreciation charges over time, and the straight line method, which has constant depreciation charges over time. Wakeman (1980) has shown that in a situation where there is a flat tax rate and where taxable income is non-negative in all periods and for all depreciation methods under consideration, the most accelerated method is preferable for tax purposes. This is a consequence of the fact that a more accelerated method typically shifts taxable income to later periods, and when future money is discounted, paying taxes later is preferable to paying them now. Berg et al. (2000) consider a similar situation but allow for uncertainty in future cash-flows as well as a progressive tax structure. They show that a less accelerated method can then be optimal.

The above described literature on optimal tax depreciation considers a static situation where a given value has to be depreciated over a maximum number of periods. In practice, most firms regularly engage in replacement investments and adjust their depreciation policy accordingly. Moreover, many countries do not use a flat tax rate in corporate taxation. The focus of this paper therefore is on optimal tax depreciation given reinvestments and a progressive tax system. ${ }^{1}$ In order to study the effects of the tax system on the optimal depreciation charges, we focus on a firm in a steady state with respect to its investment policy, i.e. the firm makes replacement investments in order to compensate for technical deterioration, so that its capital stock remains constant. ${ }^{2}$

The main results are as follows. The firm reaches a steady state with respect to

\footnotetext{
${ }^{1}$ For a dynamic model where the depreciation policy is taken as given and the firm optimizes over its investment policy, see Wielhouwer et al. (2000) .

${ }^{2}$ For all dynamic models of the firm (see e.g. Van Hilten et al. (1993)), the typical final situation is a steady state, where only replacement investments are made.
} 
tax depreciation after a finite number of periods. In the steady state the depreciation charge equals the amount that is reinvested. Before the steady state is reached, the optimal policy trades off the benefits of accelerated depreciation and of constant depreciation. Accelerated depreciation shifts taxable income to later periods, but constant depreciation can lead to a lower marginal tax rate. Therefore, contrary to what is often believed, the most accelerated method is not necessarily the best from a tax perspective, and this even holds if taxable income is always positive. From a technical point of view it is interesting that the path coupling procedure, which is mostly used for solving continuous time dynamic models, is now applied within a discrete time setting.

This paper is organized as follows. In Section 2 the model is formulated and the necessary conditions are derived. The model is solved in Section 3 by using the path coupling method. Readers that are less interested in technical details can skip Section 3 and move on directly to Section 4, where the results are economically analyzed.

\section{The model}

Consider a firm in a steady state with a capital stock $K$. The firm produces with this capital stock, and revenue is a function $C(\cdot)$ of $K$. Since the firm is in a steady state, revenue is constant and equals, say, $C$ in all periods.

The initial tax base of assets, which is the initial amount to be depreciated in the future periods, is denoted by $\tilde{D}$. Furthermore, let $D_{i}$ be the tax base at the beginning of period $i+1$. Hence, according to this notation $D_{0}=\tilde{D}$. The control variable (tax depreciation rate) in period $i$ is the fraction $\gamma_{i} \in[0,1]$ of $D_{i-1}$ depreciated in period $i$. This implies that the amount $d_{i}$ depreciated in period $i$ equals $\gamma_{i} D_{i-1}$.

Apart from the tax depreciation, there is also depreciation that reflects technical deterioration, which is constant in the steady state. In order to remain in this steady state the firm carries out replacement investments $I$, which equal the amount of technical depreciation. In this way capital stock remains constant over time. 
However, investments not only affect the capital stock. The tax base of assets also increases with $I$. Here it is important to note that the development of the tax base is unaffected by technical depreciation, but instead depends on tax depreciation. This results in the following dynamics and constraints:

$$
\begin{aligned}
& D_{i}=\left(1-\gamma_{i}\right) D_{i-1}+I, \quad \text { for } i \in \mathbb{N} \backslash\{0\}, \\
& D_{0}=\tilde{D}, \\
& \gamma_{i} \in[0,1], \quad \text { for } i \in \mathbb{N} \backslash\{0\} .
\end{aligned}
$$

Since the firm is in a steady state with respect to investments, and since the amount reinvested in each period equals $I$, it holds that $\tilde{D}>I$. Moreover, it is assumed that in the steady state a positive amount of dividend is paid, so that the constants $C$ and $I$ are related in the following way:

$$
C>I \text {. }
$$

Finally the tax system is defined. A progressive tax system most often consists of several tax brackets. To keep the optimization problem tractable, we approximate this discontinuous function by a twice differentiable increasing function $\tau($.). The function should yield non-negative taxes for positive income, and no taxes for negative income. The marginal rate should be increasing but less than 1 (otherwise tax payments would exceed taxable income). Therefore we consider tax functions that satisfy:

$$
\begin{aligned}
& \tau(x)= \begin{cases}0 & \forall x \leq 0, \\
>0 & \forall x>0,\end{cases} \\
& \tau(x)<x, \\
& \tau^{\prime}(x)>0, \quad \forall x>0, \\
& \tau^{\prime}(0)=0, \\
& \tau^{\prime \prime}(x)>0, \quad \forall x>0 .
\end{aligned}
$$

From this specification it follows that

$$
\tau^{\prime}(x)<1 .
$$


Now, since taxable income in period $i$ equals revenue $C$ minus tax depreciation $\gamma_{i} D_{i-1}$, the taxes paid in period $i$ equal:

$$
\tau\left(C-\gamma_{i} D_{i-1}\right)
$$

The objective is to minimize the present value of all tax payments. ${ }^{3}$ In order to properly compare the value of tax payments in different periods, we introduce the discount factor $\alpha=\frac{1}{1+\text { interest rate }}$ with $\alpha \in(0,1)$. The optimization problem then is:

$$
\begin{array}{r}
\min _{\gamma_{i}} \sum_{i=1}^{\infty} \alpha^{i} \tau\left(C-\gamma_{i} D_{i-1}\right) \\
\text { s.t. (1), (2) and (3). }
\end{array}
$$

\section{Solution of the Control Problem}

When solving the model with the path coupling method, the following steps will be made: (1) Specify the necessary conditions, (2) specify and analyze the paths, and (3) determine the optimal sequence of paths.

\subsection{The necessary conditions}

In order to derive the necessary conditions, we formulate the current value Hamiltonian (see for details Feichtinger and Hartl (1986), pp. 504-509) with $\lambda$ as the current value costate (adjoint) variable. After replacing the min in (11) with the $\max$ of the negative, we obtain

$$
H(D, \gamma, \lambda)=-\tau(C-\gamma D)+\lambda[(1-\gamma) D+I]
$$

with the accompanying Lagrangian:

$$
L\left(D, \gamma, \lambda, \eta_{1}, \eta_{2}\right)=-\tau(C-\gamma D)+\lambda[(1-\gamma) D+I]+\eta_{1} \gamma+\eta_{2}(1-\gamma)
$$

\footnotetext{
${ }^{3}$ Notice that, when all revenue (after tax and replacement investment) is paid out to the shareholders as dividends, this is equivalent to maximizing shareholder value.
} 
The necessary conditions, for all $i \in I N \backslash\{0\}$, are:

$$
\begin{aligned}
& L_{\gamma}=\tau^{\prime}\left(C-\gamma_{i} D_{i-1}\right) D_{i-1}-\lambda_{i} D_{i-1}+\eta_{1 i}-\eta_{2 i}=0, \\
& \lambda_{i-1}=\alpha L_{D}=\alpha \tau^{\prime}\left(C-\gamma_{i} D_{i-1}\right) \gamma_{i}+\lambda_{i}\left(1-\gamma_{i}\right) \alpha \\
& D_{i}=\left(1-\gamma_{i}\right) D_{i-1}+I \\
& \eta_{1 i} \gamma_{i}=0 \\
& \eta_{2 i}\left(1-\gamma_{i}\right)=0 \\
& \eta_{j i} \geq 0, \text { for } j=1,2 \\
& \gamma_{i} \in[0,1] .
\end{aligned}
$$

\subsection{The paths}

The different policies the firm can apply follow from the complementary slackness conditions (17), (18) and (19), and they are presented in the following table:

\begin{tabular}{|c|c|c|c|c|}
\hline & 1 & 2 & 3 & 4 \\
\hline$\eta_{1}$ & $=0$ & $>0$ & $=0$ & $>0$ \\
$\eta_{2}$ & $=0$ & $=0$ & $>0$ & $>0$ \\
\hline
\end{tabular}

Clearly path 4 is not feasible in an arbitrary period since $\eta_{1}>0$ and $\eta_{2}>0$ imply that $\gamma=0$ and $\gamma=1$ at the same time. Now the necessary conditions for the three resulting paths are analyzed.

Path 1: $\eta_{1}=\eta_{2}=0$

The policy of the firm on this path is to depreciate a fraction $\gamma \in[0,1]$ that is determined by the discount rate and the tax system, but not by the constraints. Equation (14) now becomes for a period $i$ :

$$
\tau^{\prime}\left(C-\gamma_{i} D_{i-1}\right) D_{i-1}-\lambda_{i} D_{i-1}=0
$$

Since $D_{i-1} \geq I>0$, this implies that:

$$
\lambda_{i}=\tau^{\prime}\left(C-\gamma_{i} D_{i-1}\right)
$$


Based on this expression an intuitive explanation can be given in terms of marginal revenue and marginal cost with respect to the objective function. When a fraction $\gamma_{i}$ is depreciated, the marginal revenue of depreciation is $\tau^{\prime}\left(C-\gamma_{i} D_{i-1}\right)$. The marginal 'cost' of depreciating is the shadowprice $\lambda_{i}$, which reflects the loss of value to the firm due to a marginal decrease of the tax base. Now depreciation is chosen in such a way that marginal revenue and cost are exactly equal, if this is possible with a $\gamma$ in the range $[0,1]$.

From (22) we can also conclude that tax depreciation, $\gamma_{i} D_{i-1}$, decreases as a function of $\lambda_{i}$, since $\tau($.$) is increasing. Moreover, together with (15), (22) implies$ that

$$
\lambda_{i-1}=\alpha \lambda_{i}
$$

Hence, on Path 1, $\lambda$ increases over time, which with (22) implies that tax depreciation decreases on this path.

As we will see later on, and as is intuitively clear, the shadow price of an extra unit to depreciate is positive. Then the optimal depreciation rate $\tilde{\gamma}_{i}$ on path 1 is given by:

$$
\tilde{\gamma}_{i}=\frac{C-\tau^{\prime-1}\left(\lambda_{i}\right)}{D_{i-1}} .
$$

Path 2: $\eta_{1}>0, \eta_{2}=0$

From (17) it is obtained that on this path the policy is to depreciate nothing, i.e. $\gamma=$ 0 . Due to (15) it is found immediately that the dynamics of the co-state is the same as on Path 1, so (23) holds. Since nothing is depreciated, the tax base increases with $I$. Finally, (14) implies that

$$
\tau^{\prime}(C) D_{i-1}-\lambda_{i} D_{i-1}+\eta_{1}=0
$$

Since $\eta_{1}>0$ on this path, it follows that:

$$
\lambda_{i}>\tau^{\prime}(C)
$$


Apparently, the marginal cost of depreciation $\left(\lambda_{i}\right)$ exceeds marginal revenue $\left(\tau^{\prime}(C)\right)$, so that the firm would optimally like to have a negative depreciation. Since depreciation is restricted to be non-negative, the second best thing to do is to depreciate nothing, and therefore the optimal $\gamma$ equals zero.

Path 3: $\eta_{1}=0, \eta_{2}>0$

Together with (18), $\eta_{2}>0$ implies that on this path the policy is to depreciate as much as possible, i.e. $\gamma=1$. From (15) one finds the dynamics of the co-state when Path 3 is applied in period $i$ :

$$
\lambda_{i-1}=\alpha \tau^{\prime}\left(C-D_{i-1}\right)
$$

From expression (1), it can be concluded that after using this policy in one period, the tax base in the next period will be $I$. Furthermore, (14) leads to:

$$
\tau^{\prime}\left(C-D_{i-1}\right) D_{i-1}-\lambda_{i} D_{i-1}-\eta_{2}=0
$$

and, given that $\eta_{2}>0$ on this path, it follows that:

$$
\lambda_{i}<\tau^{\prime}\left(C-D_{i-1}\right)
$$

Hence, in order to find a balance between current revenue of depreciation and future revenues that are foregone by depreciating now, the firm would optimally like to depreciate more than the tax base. This is a consequence of the fact that the increase in marginal tax rate in future periods does not compensate the gain that can be achieved by deferring taxes to future periods. Therefore, the second best thing to do is to depreciate the whole tax base.

\subsection{The optimal solution}

After having established the characteristics of the paths, the next step is to derive the optimal sequence. In this section, first all sequences of paths that satisfy the necessary optimality conditions are determined in Proposition 3.2. This results in a number of candidate optimal solutions. In Proposition 3.3 it is shown which one of 
them is optimal.

Lemma 3.1 Path 2 will never occur in an optimal solution.

Proof: Since it is clear that following Path 2 from a certain period on until infinity is suboptimal, it is sufficient to show that if Path 2 is applied in period $i$, it cannot be followed by Path 1 or Path 3 in period $i+1$.

Now suppose that Path 2 is applied in period $i$. Then it follows from (14) and (15) that:

$$
\lambda_{i}>\tau^{\prime}(C)
$$

Now, let Path 3 be applied in period $i+1$. Then it follows from (15) that for period $i+1$ it holds that:

$$
\lambda_{i}=\alpha \tau^{\prime}\left(C-D_{i}\right)<\tau^{\prime}(C)
$$

which is contradictory to $(26)$.

Now, let Path 1 be applied in period $i+1$. From (22) and (23) it is obtained that for period $i+1$ we have:

$$
\lambda_{i}=\alpha \lambda_{i+1}=\alpha \tau^{\prime}\left(C-\gamma_{i+1} D_{i}\right)
$$

Then again this leads to a contradiction with (26), since

$$
\lambda_{i}=\alpha \tau^{\prime}\left(C-\gamma_{i+1} D_{i}\right)<\tau^{\prime}(C)
$$

This completes the proof.

In the next lemma it is shown that, once it is optimal for the firm to depreciate the whole tax base (reinvestments of the last period plus the residual tax base) in a given period, it is optimal to depreciate the replacement investments at once in all the following periods. In terms of the model this means that, if $\gamma_{i}=1$ is optimal in period $i$, it is optimal to have $\gamma_{k}=1$ in all later periods. 
Lemma 3.2 When at time $i$ it holds that $\gamma_{i}=1$ is optimal, then $\gamma_{k}=1$ is optimal for all periods $k \geq i$.

Proof: In the proof, we will subsequently show the following:

A) depreciation decreases when Path 3 is applied immediately after Path 1;

B) depreciation decreases when Path 1 is applied in subsequent periods;

C) the statement in this lemma, using A) and B).

A) When Path 1 is applied in period $i$ and Path 3 in period $i+1$ it holds that:

$$
\begin{aligned}
& \text { period } i \rightarrow \lambda_{i}=\tau^{\prime}\left(C-\gamma_{i} D_{i-1}\right), \\
& \text { period } i+1 \rightarrow \lambda_{i}=\alpha \tau^{\prime}\left(C-D_{i}\right) .
\end{aligned}
$$

This implies that

$$
\tau^{\prime}\left(C-\gamma_{i} D_{i-1}\right)<\tau^{\prime}\left(C-D_{i}\right) .
$$

With $\tau^{\prime \prime}(\cdot)>0$ it follows that $\gamma_{i} D_{i-1}>D_{i}$.

B) Already proved while presenting Path 1 in Section 3.2.

C) We will show that if $\gamma_{i}=1$ in the optimal solution, then Path 3 is optimal in all periods $k \geq i+1$.

We know from lemma 3.1 that Path 2 is never optimal. Now suppose that in an optimal solution $\gamma_{i}=1$, and Path 1 is applied in period $i+1$. Then, since the depreciation charge in period $i$ equals $D_{i-1}$, it follows from (1) that the maximal depreciation charge in period $i+1$ is $D_{i}=I$.

Now, A) and B) imply that the optimal depreciation charges in all periods $k \geq i+2$ would be strictly less than $I$. This is clearly suboptimal since this policy is strictly dominated by the policy were $\gamma_{k} D_{k-1}=I$ for all $k \geq i+2$, since the latter yields a strictly lower present value of future tax payments. We have therefore shown that it is never optimal to apply Path 1 immediately after a period where $\gamma=1$. Since on Path 3, one has $\gamma=1$, this completes the proof. 
The following proposition states that the firm always enters a steady state with respect to the tax base at a finite point of time. At this steady state it holds that $\gamma_{i}=1$ and $D_{i}=I$.

Proposition 3.1 The optimal strategy is to apply Path 1 until a certain period J, and Path 3 from thereon.

Proof: Application of Path 3 implies that $\gamma=1$, so that Lemma 3.1 and Lemma 3.2 imply that it is sufficient to show that Path 1 cannot be followed optimally until infinity.

Using Path 1 from period 0 until infinity implies that

$$
\left(\frac{1}{\alpha}\right)^{i-1} \lambda_{0}=\alpha \lambda_{i}=\lambda_{i-1} \leq \tau^{\prime}(C)
$$

so, given that $\lambda_{0}$ is positive ${ }^{4}$, it follows immediately that this inequality cannot be satisfied for arbitrary large $i$.

Now, the only thing that is left to do is to determine the optimal $J$ and the optimal fractions $\gamma_{1}, \ldots, \gamma_{J-1}$. The next proposition restricts the possible values of $J$. Before presenting this proposition, we first need the following lemma.

Lemma 3.3 Let $\Omega(.,):. \mathbb{R}_{+} \times I N \backslash\{0\} \rightarrow \mathbb{R}$ be defined as follows:

$$
\Omega(d, k)=\tilde{D}+(k-1) I-d-\sum_{i=1}^{k-1}\left[C-\tau^{\prime-1}\left(\alpha^{k-i} \tau^{\prime}(C-d)\right)\right]
$$

Then, for any $k \in I N \backslash\{0\}$, the function $\Omega(., k)$ is strictly decreasing and has exactly one root. The root of $\Omega(., k)$ will be denoted $\hat{d}_{k}$.

Proof: Trivial.

\footnotetext{
${ }^{4}$ From $(22)$ it is obtained that $\lambda$ is non-negative on Path 1 . Furthermore, on Path 1 it holds that $\alpha \lambda_{i}=\lambda_{i-1}$. So if $\lambda_{0}=0$, then $\lambda_{i}=0$ for all $i$ in case path 1 is applied until infinity. Then, (14) implies that $D_{i-1}=0$ or $\gamma_{i} D_{i-1}>C$ for all $i$. The latter implies that $D_{i-1}-D_{i}>C$ as long as $D_{i-1}>0$. This implies that eventually the tax base becomes zero or negative in both cases, which is contradictory to $(1)$ and (20). Hence, $\lambda_{0}$ must be positive.
} 
The interpretation is as follows. Consider a depreciation scheme that enters the steady state in period $J$. In Proposition 3.2 we will show that when $d$ equals the depreciation amount in period $J$, the last $J-1$ terms of $\Omega(d, J)$ represent the depreciation charges in periods $1, \ldots, J-1$, as a function of $d$. Consequently, since the first two terms of $\Omega(d, J)$ represent the maximal amount that can be depreciated until period $J$, a solution where the steady state is reached in period $J$ can only be feasible if $\Omega(d, J)=0$.

The following proposition characterizes the set of candidate optimal depreciation strategies, i.e. those strategies that satisfy the necessary conditions for optimality (14)-(20).

Proposition 3.2 Let $\hat{d}_{J}$ be the root of $\Omega(., J)$. Then, the optimal strategy has the following form:

$$
\begin{array}{ll}
\gamma_{i}=\frac{C-\tau^{\prime-1}\left(\alpha^{J-i} \tau^{\prime}\left(C-D_{J-1}\right)\right)}{D_{i-1}}, & \text { for } i=1, \ldots, J-1, \\
\gamma_{i}=1, & \text { for } i=J, \ldots, \infty .
\end{array}
$$

where

$$
D_{J-1}=\hat{d}_{J}
$$

for some $J$ that satisfies $\hat{d}_{J} \in[I, \tilde{D}]$.

Proof: Proposition 3.1 implies that there exists a period $J$ such that $\gamma_{k}=1$ for all $k \geq J$, and $\gamma_{k}<1$ for all $k<J$. Therefore, (29) is satisfied.

Since Path 1 is applied before $J$ with $\gamma<1$, it follows that in period $J(25)$ holds, while in period $J-1$ we have (23). This enables us to solve the difference equation for the costate and we find

$$
\begin{aligned}
& \lambda_{i}=\alpha^{J-i} \tau^{\prime}\left(C-D_{J-1}\right), \quad \text { for } i=0, \ldots, J-1, \\
& \lambda_{i}=\alpha \tau^{\prime}(C-I), \quad \text { for } i=J, \ldots, \infty
\end{aligned}
$$


Note that the costate variables in (31) are functions of $D_{J-1}$. From these costate variables, the $\gamma_{i}$ can be determined, also as a function of $D_{J-1}$, using (24). This yields (28).

Subsequent substitution of the $\gamma_{i}^{\prime}$ 's in (16) leads to $\Omega\left(D_{J-1}, J\right)=0$, so that $D_{J-1}=$ $\hat{d}_{J}$, and (30) is satisfied. Notice that this implies that the amounts depreciated in periods 1 until $J$ equal the amount that was initially to be depreciated plus all reinvestments in these periods.

Finally, we show that $\hat{d}_{J} \in[I, \tilde{D}]$. From (20), (16), and (30) it follows immediately that $\hat{d}_{J}=D_{J-1} \geq I$. Given that the maximum possible amount of depreciation in period 1 equals $\tilde{D}$, that depreciation charges decrease on Path 1 , that depreciation decreases when going from Path 1 to Path 3, and that the depreciation charge in period $J$ equals $d_{J}=D_{J-1} \geq I$, it follows immediately that

$$
D_{J-1}=\tilde{D}+(J-1) I-\sum_{k=1}^{J-1} d_{k} \leq \tilde{D}
$$

so that a feasible solution arises when $D_{J-1}=\hat{d}_{J} \in[I, \tilde{D}]$. Calculating backwards using this $D_{J-1}$ yields a depreciation scheme that satisfies all necessary conditions.

The above proposition implies that the optimal strategy will reach the steady state in a period $J$ that satisfies $\hat{d}_{J} \in[I, \tilde{D}]$, where $\hat{d}_{J}$ is the unique root of $\Omega(., J)$. Moreover, it yields the optimal depreciation charges in all periods before the steady state, as a function of $J$ and $\hat{d}_{J}$. In general, there will be multiple $J$ 's that satisfy $\hat{d}_{J} \in[I, \tilde{D}]$, so that it remains to determine the optimal $J$. It will turn out that it is optimal to use the strategy where $\gamma=1$ is postponed as long as possible, i.e. $J$ is maximal. Therefore, contrary to what is often thought, depreciating more slowly strictly dominates a more accelerated depreciation scheme. Before this result can be mathematically derived, we first study the set of periods $J$ that yield a candidate optimal strategy.

Lemma 3.4 Let $Z \subset I N \backslash\{0\}$ be defined as follows:

$$
Z:=\left\{k \in \mathbb{I} \backslash\{0\} \mid \hat{d}_{k} \in[I, \tilde{D}]\right\} .
$$


The set $Z$ has the following properties:

i) $Z$ is non-empty.

ii) $\hat{d}_{k}$ is decreasing in $k$ for all $k \in Z$.

iii) If $i \in Z$, then $k \in Z$ for all $k \leq i$,

iv) $k \in Z \Leftrightarrow \Omega(I, k) \geq 0$.

v) $Z$ is finite, and

$$
k \in Z \Rightarrow k \leq \frac{\tilde{D}-I}{\delta}+1
$$

where $\delta=C-I-\left(\tau^{\prime}\right)^{-1}\left(\alpha \tau^{\prime}(C-I)\right)$.

Proof: $i$ ) Follows immediately from the fact that $\Omega(\tilde{D}, 1)=0$, so that $\hat{d}_{1}=\tilde{D}$ and $1 \in Z$.

ii) Evaluating $\Omega(\cdot, k+1)$ in $\hat{d}_{k}$ for some $k \in Z$ yields:

$$
\begin{aligned}
\Omega\left(\hat{d}_{k}, k+1\right) & =\Omega\left(\hat{d}_{k}, k\right)+I-\left[C-\tau^{\prime-1}\left(\alpha^{k} \tau^{\prime}\left(C-\hat{d}_{k}\right)\right)\right] \\
& =I-\left[C-\tau^{\prime-1}\left(\alpha^{k} \tau^{\prime}\left(C-\hat{d}_{k}\right)\right)\right]
\end{aligned}
$$

Since

$$
C-\tau^{\prime-1}\left(\alpha^{k} \tau^{\prime}\left(C-\hat{d}_{k}\right)\right)>C-\tau^{\prime-1}\left(\tau^{\prime}\left(C-\hat{d}_{k}\right)\right)=\hat{d}_{k} \geq I
$$

one has that:

$$
\Omega\left(\hat{d}_{k}, k+1\right)<0
$$

Therefore, since $\frac{\partial \Omega(d, k+1)}{\partial d}<0$, it holds that $\hat{d}_{k+1}<\hat{d}_{k}$.

iii) Together with $\left.\hat{d}_{1}=\tilde{D}, i i\right)$ implies that

$$
k \in Z \Leftrightarrow \hat{d}_{k} \geq I
$$

It now follows immediately from $i i$ ) and (35) that $k+1 \in Z$ implies that $k \in Z$. 
$i v$ ) Since $\Omega(., k)$ is strictly decreasing for all $k$, it follows from (35) that

$$
k \in Z \Leftrightarrow \Omega(I, k) \geq 0 .
$$

$v$ ) Let us denote

$$
\delta:=C-I-\left(\tau^{\prime}\right)^{-1}\left(\alpha \tau^{\prime}(C-I)\right) .
$$

Then it follows from the convexity of $\tau($.$) and the fact that \alpha<1$ that $\delta>0$. Moreover, for any $j \geq 1$, one has:

$$
C-I-\left(\tau^{\prime}\right)^{-1}\left(\alpha^{j} \tau^{\prime}(C-I)\right) \geq \delta .
$$

This implies that

$$
\begin{aligned}
\Omega(I, k) & =\tilde{D}-I-\sum_{i=1}^{k-1}\left(C-I-\left(\tau^{\prime}\right)^{-1}\left(\alpha^{k-i} \tau^{\prime}(C-I)\right)\right) \\
& \leq \tilde{D}-I-(k-1) \delta .
\end{aligned}
$$

Therefore,

$$
\Omega(I, k) \geq 0 \Rightarrow k \leq \frac{\tilde{D}-I}{\delta}+1,
$$

so that it follows from $i i$ ) that $Z$ is finite.

This concludes the proof.

Notice that the fact that $Z$ is finite implies that an optimum exists. Indeed, for every $J \in Z$ there is a candidate optimal strategy as given in Proposition 3.2, and the optimal strategy is the one in which total discounted tax payments are minimal. In the following proposition we show that, in comparing the present value of future tax payments for two potential optimal solutions as given in Proposition 3.2 , the solution with the highest value of $J$ yields the lowest discounted future tax payments. This immediately implies that the optimal strategy is the one in which $\gamma=1$ is postponed as long as possible. 
Proposition 3.3 An optimal depreciation scheme exists, and it is given by (28), (29) and (30) for

$$
J=\max \{k \mid \Omega(I, k) \geq 0\} .
$$

Proof: The fact that an optimum exists follows immediately from Proposition 3.2 and Lemma $3.4 v)$.

Now, for any $J \in I N \backslash\{0\}$, we denote $\operatorname{Tax}(J)$ for the discounted future tax payments for the candidate optimal solution that satisfies (28), (29) and (30). Similarly, we denote $\gamma_{i}^{J}$, and $D_{i-1}^{J}$ for the corresponding depreciation fractions and residual tax base in period $i$.

Given Proposition 3.2 and Lemma $3.4 i v$ ) and $v$ ), it suffices to show that for any $J, M \in Z$ with $M<J$, it holds that $\operatorname{Tax}(J)<\operatorname{Tax}(M)$.

Now, take any $M<J \in Z$. Then,

$$
\begin{aligned}
& \operatorname{Tax}(J)-\operatorname{Tax}(M) \\
&= \sum_{i=1}^{J} \alpha^{i-1} \tau\left(C-\gamma_{i}^{J} D_{i-1}^{J}\right)-\sum_{i=1}^{M} \alpha^{i-1} \tau\left(C-\gamma_{i}^{M} D_{i-1}^{M}\right)-\sum_{i=M+1}^{J} \alpha^{i-1} \tau(C-I) \\
&= \sum_{i=1}^{M} \alpha^{i-1}\left[\tau\left(C-\gamma_{i}^{J} D_{i-1}^{J}\right)-\tau\left(C-\gamma_{i}^{M} D_{i-1}^{M}\right)\right] \\
& \quad+\sum_{i=M+1}^{J} \alpha^{i-1}\left[\tau\left(C-\gamma_{i}^{J} D_{i-1}^{J}\right)-\tau(C-I)\right] \\
&< \sum_{i=1}^{M} \alpha^{i-1} \tau^{\prime}\left(C-\gamma_{i}^{J} D_{i-1}^{J}\right)\left[\gamma_{i}^{M} D_{i-1}^{M}-\gamma_{i}^{J} D_{i-1}^{J}\right] \\
& \quad+\sum_{i=M+1}^{J} \alpha^{i-1} \tau^{\prime}\left(C-\gamma_{i}^{J} D_{i-1}^{J}\right)\left[I-\gamma_{i}^{J} D_{i-1}^{J}\right]
\end{aligned}
$$

where the inequality follows immediately from the convexity of $\tau($.$) . Now, it follows$ immediately from (29) that

$$
\begin{aligned}
\gamma_{i}^{J} D_{i-1}^{J} & =C-\tau^{\prime-1}\left(\alpha^{J-i} \tau^{\prime}\left(C-D_{J-1}^{J}\right)\right), \\
\gamma_{i}^{M} D_{i-1}^{M} & =C-\tau^{\prime-1}\left(\alpha^{M-i} \tau^{\prime}\left(C-D_{M-1}^{M}\right)\right) .
\end{aligned}
$$


Therefore, it follows that:

$$
\begin{aligned}
& \operatorname{Tax}(J)-\operatorname{Tax}(M) \\
&<\sum_{i=1}^{M} \alpha^{i-1} \alpha^{J-i} \tau^{\prime}\left(C-D_{J-1}^{J}\right)\left[\gamma_{i}^{M} D_{i-1}^{M}-\gamma_{i}^{J} D_{i-1}^{J}\right] \\
&+\sum_{i=M+1}^{J} \alpha^{i-1} \alpha^{J-i} \tau^{\prime}\left(C-D_{J-1}^{J}\right)\left[I-\gamma_{i}^{J} D_{i-1}^{J}\right] \\
&=-\alpha^{J-1} \tau^{\prime}\left(C-D_{J-1}^{J}\right) \sum_{i=1}^{J} \gamma_{i}^{J} D_{i-1}^{J}+\alpha^{J-1} \tau^{\prime}\left(C-D_{J-1}^{J}\right)\left[\sum_{i=1}^{M} \gamma_{i}^{M} D_{i-1}^{M}+\sum_{i=M+1}^{J} I\right]I] \\
&=-\alpha^{J-1} \tau^{\prime}\left(C-D_{J-1}^{J}\right)[\tilde{D}+(J-1) I] \\
& \quad+\alpha^{J-1} \tau^{\prime}\left(C-D_{J-1}^{J}\right)[\tilde{D}+(M-1) I+(J-M) I] \\
&=0 .
\end{aligned}
$$

This completes the proof.

\section{Economic Analysis}

We study a situation in which the firm has grown up, i.e. capital stock has reached its long term optimal level so that investment is fixed in such a way that capital stock remains constant at this level. The first implication is that the firm's revenue is constant over time. The second implication is that, in the absence of depreciation, the tax base of assets increases each period with the same investment level. The problem that we focus on in this paper is how the firm should fix its depreciation schedule such that its discounted tax payments are minimized, given that the tax system is progressive. A progressive tax system implies that the firm's marginal tax payments are increasing with net revenue, where net revenue is defined as the difference between revenue (which is constant here) and the amount depreciated. The restriction is that for a given time period depreciation is bounded from below by zero. On the other hand tax payments are only increasing with net revenue as long as net revenue is non-negative, i.e. in case of negative net revenue the firm does 
not receive any money from the government. The implication for optimal behavior is that the decision maker will fix its amount depreciated such that for each period this amount will never exceed the firm's revenue.

While determining the amounts depreciated two major effects can be distinguished: the discounting effect and the progressive tax effect. Let us first elaborate on the discounting effect. To look at this effect in isolation replace for the moment the progressive tax system by a linear one so that, independent of the level of net revenue, the same amount of tax need to be paid on an additional unit of net revenue. Due to discounting the periods are weighed in such a way that larger weights are assigned to earlier periods. Consequently, an optimal depreciation schedule will accomplish that tax payments are minimized in the first period, which can be achieved by depreciating as much as possible in the first period. For the case that the tax base (including the investment expenditure of the first period) falls below the per period revenue, i.e. $\tilde{D}<C$, this implies that the total tax base is depreciated in the first period, while in the periods after that, the investment expenditure $I$ is depreciated immediately. In case $\tilde{D}>C$ the amount $C$ will be depreciated in the first period (as remarked above it makes no sense to depreciate more than $C$ ). The implication is that net revenue equals zero so that the amount of tax paid in period 1 equals zero too. Then at the beginning of the second period the tax base equals $D_{1}=\tilde{D}-C+I$. In case $D_{1}<C$ the total tax base will be depreciated, otherwise the firm depreciates $C$ resulting again in zero tax payments. And so on and so forth.

To study the progressive tax effect in isolation assume for the moment that $\alpha$ is very close to 1 , so that almost no discounting takes place. Since the tax function $\tau($.) is convex, tax payments are minimized if the firm depreciates in such a way that net revenue is equal for all periods. Since revenue is constant over time it follows that also the amount depreciated is constant. In each period the tax base increases with the investment expenditure, and therefore the amount depreciated will be at least as high as $I$. More precisely, the amount depreciated converges to the sum of 
the investment expenditures from period 1 onwards plus the initial tax base $(\tilde{D})$, divided by the amount of time periods considered. Because our planning period has infinite length, it follows that the amount depreciated converges to $I$ in all periods. Formally, we have the following result.

Proposition 4.1 Let $J(\alpha)$ denote the period in which the steady state is reached and let $d_{i}(\alpha)$ denote the depreciation charge in period $i$ in the optimal solution, both as a function of $\alpha$. Then,

$$
\begin{aligned}
& \lim _{\alpha \rightarrow 1} J(\alpha)=\infty, \\
& \lim _{\alpha \rightarrow 1} d_{i}(\alpha)=I, \quad \text { for all } i \in I N .
\end{aligned}
$$

Proof: Since our aim is to study the effect of $\alpha$ converging to 1 , we now consider $\alpha$ being variable, and therefore we denote $\Omega(I, k, \alpha)$ for the function as defined in Lemma 3.3.

It is seen immediately that

$$
\lim _{\alpha \rightarrow 1} \Omega(I, k, \alpha)=\tilde{D}-I>0, \quad \text { for all } k \text {. }
$$

Therefore it follows that, for all $k$, there exists an $\alpha_{k} \in[0,1)$ such that $\Omega\left(I, k, \alpha_{k}\right)>$ 0 , which implies that $J\left(\alpha_{k}\right)>k$. As a consequence we can conclude that

$$
\sup _{\alpha \in[0,1)} J(\alpha)=\infty
$$

Now, since $J(\cdot)$ is increasing in $\alpha$ (see Proposition 4.3) this implies that

$$
\lim _{\alpha \rightarrow 1} J(\alpha)=\infty
$$

In order to show (41), notice that

$$
\Omega(d, k, 1)=\tilde{D}-d-(k-1)(d-I), \quad \text { for all } k
$$

Therefore, the root of $\Omega(., k, 1)$ equals $\frac{\tilde{D}+(k-1) I}{k}$. 
Now, since Proposition 3.2 implies that in the optimal solution the depreciation charge in period $J(\alpha)$ (denoted $d_{J(\alpha)}(\alpha)$ ) is given by the root of $\Omega(\cdot, J(\alpha), \alpha)$ (denoted $\hat{d}_{J(\alpha)}(\alpha)$ ), it follows immediately from (43) that

$$
\lim _{\alpha \rightarrow 1} d_{J(\alpha)}(\alpha)=\lim _{k \rightarrow \infty} \frac{\tilde{D}+(k-1) I}{k}=I .
$$

Together with (28), this implies that for all $i \leq J(\alpha)$, one has

$$
\begin{aligned}
\lim _{\alpha \rightarrow 1} d_{i}(\alpha) & =\lim _{\alpha \rightarrow 1} \gamma_{i} D_{i-1} \\
& =\lim _{\alpha \rightarrow 1} D_{J-1} \\
& =\lim _{\alpha \rightarrow 1} \hat{d}_{J(\alpha)}(\alpha)=I,
\end{aligned}
$$

This concludes the proof.

In the solution of our problem the discounting effect and the progressive tax effect are combined. We saw that due to the first effect, from the first period onwards the amount depreciated is maximal but not above the revenue $C$, while the above proposition states that the second effect causes an amount depreciated that is constant over the planning period. Combining the effects leads to a solution where on an initial time interval $[0, J]$ the amount depreciated decreases over time. Then on the second time interval $(J, \infty)$ the amount depreciated exactly equals the investment rate for all time intervals. The solution is depicted in Figure 1 and we see that on the first interval the amount depreciated decreases with increasing speed.

This holds in general for cases where the third order derivative of the tax function is non-positive, as is shown in the following proposition.

Proposition 4.2 If $\tau^{\prime \prime \prime}() \leq$.0 , then depreciation charges decrease over time in an accelerated way on Path 1, i.e.

$$
\Delta d_{k}<\Delta d_{k-1}, \quad \text { for all } k \geq 2,
$$

where

$$
\Delta d_{k}=d_{k}-d_{k-1} .
$$




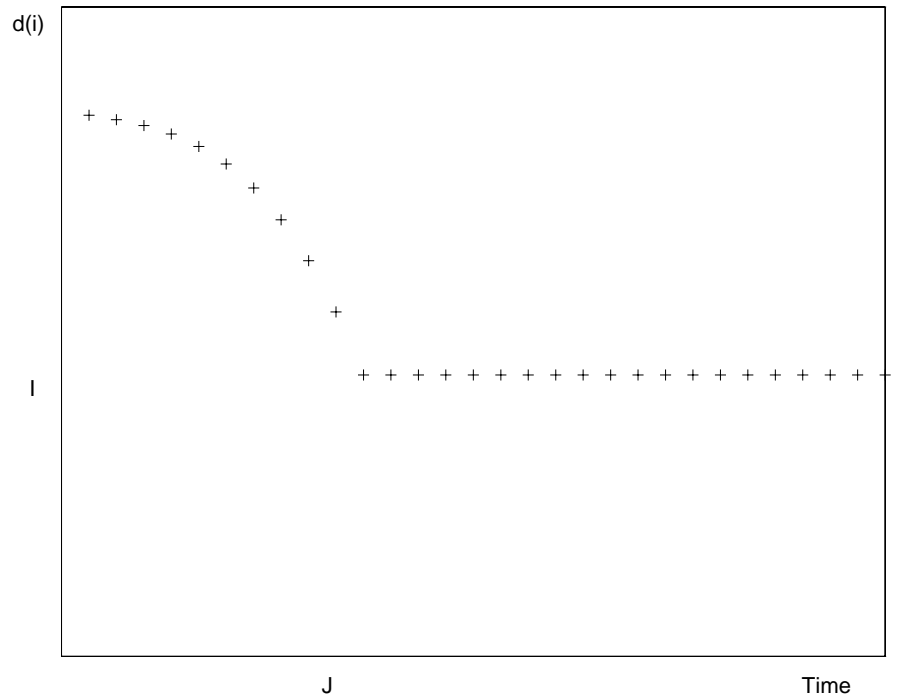

Figure 1: The optimal depreciation scheme.

Proof: Given (28) it holds that:

$$
\begin{aligned}
& \Delta d_{k}-\Delta d_{k-1} \\
= & \left(d_{k}-d_{k-1}\right)-\left(d_{k-1}-d_{k-2}\right) \\
= & -\tau^{\prime-1}\left(\alpha^{J-k} \tau^{\prime}\left(C-D_{J-1}\right)\right)+\tau^{\prime-1}\left(\alpha^{J-k+1} \tau^{\prime}\left(C-D_{J-1}\right)\right) \\
& \quad+\tau^{\prime-1}\left(\alpha^{J-k+1} \tau^{\prime}\left(C-D_{J-1}\right)\right)-\tau^{\prime-1}\left(\alpha^{J-k+2} \tau^{\prime}\left(C-D_{J-1}\right)\right) .
\end{aligned}
$$

From the convexity of $\tau^{\prime-1}($.$) , it can now be obtained that$

$$
\begin{aligned}
& \Delta d_{k}-\Delta d_{k-1} \\
& <-\left(\tau^{\prime-1}\right)^{\prime}\left(\alpha^{J-k+1} \tau^{\prime}\left(C-D_{J-1}\right)\right) \alpha^{J-k} \tau^{\prime}\left(C-D_{J-1}\right)(1-\alpha) \\
& \quad+\left(\tau^{\prime-1}\right)^{\prime}\left(\alpha^{J-k+1} \tau^{\prime}\left(C-D_{J-1}\right)\right) \alpha^{J-k+1} \tau^{\prime}\left(C-D_{J-1}\right)(1-\alpha)
\end{aligned}
$$

$<0$.

This concludes the proof.

One crucial feature of the solution is the time $J$ at which the initial time period with decreasing depreciation amounts passes into the second time interval where depreciation equals investment. In the next proposition, we show that both a lower 
discount factor or a lower initial tax base imply that the initial time period is shorter. A lower discount factor implies that the discounting effect becomes stronger relative to the tax effect, so that it makes sense that the tax base reduces at a faster rate. This results in a sooner arrival at the second time interval in which the tax base just equals the per period investment expenditure. Similarly, a lower initial tax base implies that it reaches zero sooner, so that the first time interval will be shorter.

Proposition 4.3 The period $J$ in which the steady state is reached in the optimal solution is increasing in $\alpha$ and $\tilde{D}$.

Proof: Let us again denote $\Omega(I, k, \alpha)$ for the function as defined in Lemma 3.3, and take any $\alpha_{1}<\alpha_{2}<1$.

It follows from Proposition 3.3 that

$$
\Omega\left(I, J\left(\alpha_{2}\right)+1, \alpha_{2}\right)<0
$$

Since $\Omega\left(I, J\left(\alpha_{2}\right)+1,.\right)$ is clearly increasing in $\alpha$ this implies that

$$
\Omega\left(I, J\left(\alpha_{2}\right)+1, \alpha_{1}\right)<0
$$

Now, Proposition 3.3 yields that

$$
J\left(\alpha_{1}\right) \leq J\left(\alpha_{2}\right)
$$

so that we can conclude that $J($.$) is increasing in \alpha$. The proof of the fact that $J$ is increasing in $\tilde{D}$ is similar.

In conclusion, the combined effect of time discounting and a progressive tax structure implies that the optimal solution with respect to the tax depreciation is as follows: Depreciate accelerated, balancing the time value of money and the increase in marginal tax rate, until tax depreciation equals replacement investments. Then the steady state of the tax base is reached. The firm will remain there by equating tax depreciation, technical depreciation and investments. 


\section{References}

[1] Berg, M., A. De Waegenaere, and J.L. Wielhouwer (2000), "Optimal Tax Depreciation with Uncertain Future Cash-Flows", European Journal of Operational Research, forthcoming.

[2] Feichtinger G. and R.F. Hartl (1986), Optimale Kontrolle ökonomischer Prozesse, Walter de Gruyter.

[3] van Hilten O., P.M. Kort, and P.J.J.M. van Loon (1993), Dynamic Policies of the Firm: An Optimal Control Approach, Springer, Berlijn.

[4] Scholes, M.S., And M.A. Wolfson (1992), Taxes and Business Strategy: A Planning Approach, Prentice-Hall, New Jersey.

[5] Wakeman, L.M. (1980). "Optimal Tax Depreciation". Journal of Accounting and Economics 1, 213-237.

[6] Wielhouwer, J.L, A. De Waegenaere, and P.M. Kort (2000), "Optimal Dynamic Investment Policy for Different Tax Depreciation Rates and Economic Depreciation Rates", to appear in Journal of Optimization Theory and Applications 105,2 . 